\title{
Prevalence of Vitamin D Deficiency among Perinatally HIV- Infected Thai Adolescents Receiving Antiretroviral Therapy
}

\author{
Kulkanya Chokephaibulkit, MD', Rachanee Saksawad, MD ${ }^{1}$, Torsak Bunupuradah, MD 3 , \\ Supattra Rungmaitree, MD ${ }^{1}$, Wanatpreeya Phongsamart, MD $^{1}$, Keswadee Lapphra, MD ${ }^{1}$, \\ Alan Maleesatharn, MBA $^{1}$, and Thanyawee Puthanakit, MD $^{2,3}$ \\ ${ }^{1}$ Department Of Pediatrics, Faculty Of Medicine Siriraj Hospital, Mahidol University, 2, Prannok \\ Road, Bangkok-Noi, Bangkok, Thailand, 10700 \\ ${ }^{2}$ Department Of Pediatrics, Faculty Of Medicine, Chulalongkorn University, Bangkok, Thailand \\ 10330 \\ ${ }^{3}$ HIV-NAT/Thai Red Cross AIDS Research Centre, 104 Ratchadamri Road, Pathumwan, Bangkok \\ Thailand 10330
}

\begin{abstract}
We assessed the prevalence of vitamin D deficiency among 101 perinatally HIV-infected Thai adolescents receiving antiretroviral therapy. Median age was 14.3 (IQR 13.0-15.7) years and 90\% had a HIV RNA <50 copies/mL. The median (IQR) 25-hydroxyvitamin D (25-OHD) level was $24.8(6.9-46.9) \mathrm{ng} / \mathrm{mL} ; 25$ (24.7\%) had vitamin D deficiency (25-OHD< $20 \mathrm{ng} / \mathrm{ml})$ and 47 (46.5\%) had insufficiency (25-OHD 20-30 ng/ml). Adolescents with vitamin D deficiency had significantly higher parathyroid hormone levels $(54.9$ vs. $40.2 \mathrm{pg} / \mathrm{mL}, P<0.007)$. No associations between vitamin D deficiency and body mass index, bone mineral density, efavirenz use, HIV RNA, CD4, or self-reported sunlight exposure were observed.
\end{abstract}

\section{Keywords}

Vitamin D Insufficiency; Vitamin D Deficiency; Adolescents; Human Immunodeficiency Virus; Osteopenia; Bone Health; Bone Mineral Density

\begin{abstract}
Vitamin D deficiency has been associated with multiple adverse health outcomes, including osteoporosis, cardiovascular mortality, and autoimmune disease. Several studies found high rates of vitamin D deficiency or insufficiency in HIV-infected patients, ranging from 13$77 \%^{1}$ in adults and $45-89 \%$ in children, depending on the season, latitude, and patient ethnicity. ${ }^{2,3}$ It is not known whether or how HIV infection affects vitamin D metabolism; however, it has been found that EFV and some other antiretroviral drugs do. Vitamin D is metabolized via cytochrome P450, the same pathway as many antiretroviral drugs. Several studies have reported an association between vitamin D insufficiency and non-nucleoside reverse transcriptase inhibitor (NNRTI) use, particularly with efavirenz (EFV). ${ }^{4,5}$ Low vitamin D levels have been associated with impaired immunologic responses to antiretroviral therapy (ART), HIV disease progression, and mortality. ${ }^{6}$ Although a few
\end{abstract}

Corresponding Author: Thanyawee Puthanakit, MD, Division Of Infectious Diseases, Department Of Pediatrics, Faculty Of Medicine, Chulalongkorn University, Bangkok, Thailand, 10330. Thanyawee.P@Hivnat.Org, Telephone (Fax): +66 2256-4930.

Results From This Study Were Presented In Part At The 9th International Congress Of Tropical Pediatrics, Bangkok, Thailand, October 18-20, 2011.

Disclosures: The Authors Have No Conflicts Of Interest Or Funding To Disclose. 
studies have reported a high rate of vitamin D deficiency in HIV-infected children and adolescents, data in the Asian population are limited. Tropical countries in South-East Asia may have more consistent and greater exposures to sunlight; however, current life style choices may lead to a reduction in sun exposure and may impact vitamin D status in HIVinfected adolescents in the region.

The objective of this study was to determine the prevalence of vitamin D deficiency among perinatally HIV-infected Thai adolescents receiving ART and to evaluate potential risks associated with vitamin D insufficiency.

\section{MATERIALS AND METHODS}

We performed a cross-sectional study of vitamin D levels and bone mineral density (BMD) in perinatally-HIV infected Thai adolescents aged 12-20 years receiving long-term ART at two referral centers in Bangkok: Siriraj and King Chulalongkorn Memorial hospitals. ${ }^{7}$ Subjects were evaluated for general clinical condition, tested for serum 25-hydroxyvitamin $\mathrm{D}(25(\mathrm{OH}) \mathrm{D})$, parathyroid hormone $(\mathrm{PTH})$, calcium, and underwent BMD measurement by dual-energy X-ray absorptiometry (DXA). The 25(OH)D level and PTH were measured by chemiluminescent microparticle immunoassay (ARCHITECT 25-OH vitamin D assay and ARCHITECT Intact PTH assay, Abbott Diagnostics Division, Wiesbaden, Germany). Vitamin D deficiency was defined as levels of $25(\mathrm{OH}) \mathrm{D}$ below $20 \mathrm{ng} / \mathrm{mL}$, and vitamin D insufficiency was defined as $25-\mathrm{OHD}$ between $20-30 \mathrm{ng} / \mathrm{mL}$. The upper normal limit of serum PTH was $65 \mathrm{pg} / \mathrm{mL}$. Serum calcium was measured by the VITROS Ca Slide method (Ortho-Clinical Diagnostics Inc., Rochester, New York, USA). The normal range of calcium was $8.6-10.0 \mathrm{mg} / \mathrm{dL}$. Information regarding sunlight exposure was collected by selfadministered questionnaire. Clinical data were collected from medical records, including sex, age, growth, and HIV-specific parameters, such as CD4, HIV RNA, clinical stage, and ART history.

\section{Statistical analysis}

Descriptive analysis of vitamin D levels and rates of vitamin D deficiency were reported using median and interquartile ranges (IQR). Categorical variables were compared between the adolescents with $25(\mathrm{OH}) \mathrm{D}$ level $<20 \mathrm{ng} / \mathrm{mL}$ and $>20 \mathrm{ng} / \mathrm{mL}$ using a chi-square or Fisher's Exact Test. Multivariate analyses for factors associated with vitamin D deficiency $(25(\mathrm{OH}) \mathrm{D}<20 \mathrm{ng} / \mathrm{mL})$ were performed using a forward stepwise logistic regression model that included factors with $P$-value $<0.20$ in univariate analyses, with the significant level set at $P<0.05$. Data were analyzed with SPSS Statistics 20.0.

\section{RESULTS}

\section{Demographic characteristics}

From October 2010 to February 2011, 101 perinatally HIV-infected adolescents were enrolled; 50\% male, median (IQR) age 14.3 (13.0-15.7) years. The median (IQR) duration of ART was 83.9 (52.2-104.2) months and the current ART regimens were NNRTI-based [including nevirapine (NVP) 30\%, EFV 20\%], and PI-based (50\%). Median (IQR) CD4 count was 646 (506-796) cells $/ \mathrm{mm}^{3}$, and 90\% had a plasma HIV RNA $<50 \mathrm{copies} / \mathrm{ml}$ in the previous six months. Thirty-nine (38.6\%) adolescents were in World Health Organization (WHO) clinical stage 3, and $28(27 \%)$ were in stage 4 prior to ART initiation. None of the adolescents were currently receiving vitamin $\mathrm{D}$ or calcium supplements. 


\section{Vitamin D levels and associated factors related to vitamin $D$ deficiency}

The median (IQR) 25(OH)D level was 24.8 (6.9-46.9) ng/mL. Forty-seven (46.5\%) adolescents had vitamin D insufficiency, and 25 (24.7\%) had vitamin D deficiency, three of whom had 25(OH)D $<10 \mathrm{ng} / \mathrm{ml}$. The median (IQR) PTH was 44.0 (15.4-121.6) $\mathrm{pg} / \mathrm{mL}$, and $19 \%$ had elevated PTH (>65 pg/mL). The median (IQR) calcium level was 9.8 (8.7-11.0) $\mathrm{mg} / \mathrm{dL}$ and $6 \%$ had a calcium level $>10 \mathrm{mg} / \mathrm{dL}$. The median duration of sun exposure was 7.0 (2.9-14.8) hours per week.

In a univariate analysis, adolescents with vitamin D deficiency had higher PTH levels; 54.9 (38-77) vs. 40.2 (32.6-54.9), $P=0.007$. Among 25 adolescents with vitamin $\mathrm{D}$ deficiency, $44 \%$ had high PTH levels compared to 10\% without vitamin D deficiency [6.7 (2.3-19.6), $P<0.001]$. Other factors such as sex, body mass index (BMI), BMD, ART regimen, duration of ART, HIV RNA, CD4 level, WHO staging, calcium level, and self-reported duration of sun exposure were not found to be associated with vitamin D deficiency or insufficiency. In multivariate analysis, a high PTH was the one variable associated with $25(\mathrm{OH}) \mathrm{D}<20 \mathrm{ng} / \mathrm{ml}$ (see Table, Supplemental Digital Content 1, http://links.lww.com/INF/B605).

\section{DISCUSSION}

In this report, we found that half of perinatal HIV-infected adolescents receiving ART in the greater Bangkok metropolitan area had vitamin D insufficiency and a quarter of them had vitamin D deficiency. However, there was no association between low vitamin D level and BMD. A recent study of 157HIV-uninfected Thai children between 6 to 12 years of age at our center revealed that $19 \%$ and $60 \%$ had $25(\mathrm{OH}) \mathrm{D}$ level between $10-<20$ and $20-30 \mathrm{ng} /$ $\mathrm{ml}$, respectively, but no children had a $25(\mathrm{OH}) \mathrm{D}$ level $<10 \mathrm{ng} / \mathrm{mL} .{ }^{8}$ In that cohort, low vitamin D levels were associated with higher body weight, and PTH was higher in those with vitamin D deficiency. ${ }^{8}$ A report from the Thai National Health Examination Survey in 2008-2009 of 280 healthy people aged above 15 years in Bangkok revealed vitamin D insufficiency in $65 \%$ and deficiency in $14 \% .9^{9}$ Although we found a slightly higher rate of vitamin D deficiency in HIV-infected adolescents, but overall rate of vitamin D insufficiency plus deficiency appears to be within a similar range to that seen in HIVuninfected Thai children and adults. The report in 13 US cities (REACH cohort) of high-risk adolescents 14-18 years of age that found a high rate of vitamin D deficiency (overall 87\%), but no differences between HIV-positive and HIV-negative adolescents. ${ }^{10}$ Another report in adults found a similarly high prevalence of vitamin D insufficiency or deficiency in the HIV-infected (70\%) and the general adult populations (79\%) in the US. ${ }^{4}$

Many studies have reported an association between EFV treatment and vitamin D deficiency, which may be explained by interference by EFV of the cytochrome P450 enzyme pathway involved in vitamin D metabolism. ${ }^{4,5}$ Vitamin D insufficiency was found to impair CD4 recovery in advanced HIV diseases, and associated with disease progression. ${ }^{6}$ However, vitamin D supplementation in a small study of 54 children did not improve CD4 levels, ${ }^{11}$ suggesting that the association found with vitamin $\mathrm{D}$ deficiency might be noncausal. A large study among HIV-infected adults in the US revealed similar risk factors for vitamin D insufficiency or deficiency as in HIV-uninfected individuals (i.e., Black or Hispanic ethnicity, high BMI, hypertension, lack of exercise, renal insufficiency) in addition to exposure to EFV and/or ritonavir. ${ }^{4}$ In our study, however, we did not find an association between vitamin D deficiency and BMI, bone mineral density, CD4 cell count level, HIV RNA, WHO stage, EFV use, or self-reported duration of sun exposure. The adolescents in our study were relatively healthy and had been receiving ART for a median duration of seven years. The immunologic and other body system may have been normalized and could have compensated for the HIV-related or treatment-related affects on vitamin D status. We 
found significantly higher PTH levels in adolescents with vitamin D deficiency, which likely reflects the anticipated compensatory mechanism to regulate serum calcium.

The limitations of this study included the cross-sectional design with no control group, and the study was conducted in a single urban center. These limitations preclude the generalizability of the data. However, this study provide new knowledge of vitamin D status in HIV-infected adolescents who are receiving long-term ART in Thailand. Although we were unable to identify risk factors associated with vitamin D deficiency in this population, we postulate that inadequate exposure to sunlight could have contributed to the low vitamin D levels. The 2008-2009 Thai National Health Examination Survey showed that urban populations had lower $25(\mathrm{OH}) \mathrm{D}$ levels than rural populations in every region of the country, and the lowest level was found in Bangkok. In addition to an urban lifestyle that discourages outdoor activities, air pollution was suggested to be a likely contributing factor. Preventive measures and treatment of vitamin D deficiency in HIV-infected patients should be routinely emphasized, as the effects of vitamin D deficiency in this population may be more pronounced than in healthy individuals.

\section{Supplementary Material}

Refer to Web version on PubMed Central for supplementary material.

\section{Acknowledgments}

We would like to thank participants and families who participated in the study. Funding support was provided through a grant to amfAR, The Foundation for AIDS Research, as part of the International Epidemiologic Databases to Evaluate AIDS (IeDEA; U01AI069907), by the U.S. National Institutes of Health (NIH): National Institute of Allergy and Infectious Diseases (NIAID), Eunice Kennedy Shriver National Institute Of Child Health \& Human Development (NICHD) and National Cancer Institute (NCI) and the AIDS Life Association. Dr. Puthanakit is funded in part by the National Research University Project of Commission of Higher Education and the Ratchadapiseksomphot Endowment Fund (HR 1161A-55) and the Senior Researcher Scholar, Thai Research Fund (TRF). The content of this publication is solely the responsibility of the authors and does not necessarily represent the official views of any of the institutions mentioned above.

Funding: Funding Support Provided Through A Grant To Amfar, The Foundation For AIDS Research, As Part Of The International Epidemiologic Databases To Evaluate AIDS (Iedea; U01AI069907), By The U.S. National Institutes Of Health (NIH): National Institute Of Allergy And Infectious Diseases (NIAID), Eunice Kennedy Shriver National Institute Of Child Health \& Human Development (NICHD) And National Cancer Institute (NCI) And The AIDS Life Association.

\section{References}

1. Andrea G, Giovanni P, Giulio P. Vitamin D deficiency in HIV-infected patients: a systemic review. Nutrition and dietary supplements. 2011; 3:101-111.

2. Rutstein R, Downes A, Zemel B, et al. Vitamin D status in children and young adults with perinatally acquired HIV infection. Clin Nutr. 2011; 30(5):624-8. [PubMed: 21641097]

3. Eckard AR, Judd SE, Ziegler TR, et al. Risk factors for vitamin D deficiency and relationship with cardiac biomarkers, inflammation and immune restoration in HIV-infected youth. Antivir Ther. 2012; 17(6):1069-78. [PubMed: 22894927]

4. Dao CN, Patel P, Overton ET, et al. Low vitamin D among HIV-infected adults: prevalence of and risk factors for low vitamin D Levels in a cohort of HIV-infected adults and comparison to prevalence among adults in the US general population. Clin Infect Dis. 2011; 52(3):396-405. [PubMed: 21217186]

5. Welz T, Childs K, Ibrahim F, et al. Efavirenz is associated with severe vitamin D deficiency and increased alkaline phosphatase. AIDS. 2010; 24(12):1923-8. [PubMed: 20588161]

6. Viard JP, Souberbielle JC, Kirk O, et al. Vitamin D and clinical disease progression in HIV infection: results from the EuroSIDA study. AIDS. 2011; 25(10):1305-15. [PubMed: 21522006] 
7. Puthanakit T, Saksawad R, Bunupuradah T, et al. Prevalence and risk factors of low bone mineral density among perinatally HIV-infected Thai adolescents receiving antiretroviral therapy. J Acquir Immune Defic Syndr. 2012; 61(4):477-83. [PubMed: 22918157]

8. Reesukumal K, Manonukul K, Piboonpocanun O, et al. Vitamin D insufficiency among healthy children in Bangkok, Thailand. Clinical Chemistry. 2012; 58(10):A153. Supplement.

9. Chailurkit L, Aekplakorn W, Ongphiphadhanakul B. BMC Public Health. 2011; 11:853. [PubMed: 22074319]

10. Stephensen CB, Marquis GS, Kruzich LA, et al. Vitamin D status in adolescents and young adults with HIV infection. Am J Clin Nutr. 2006; 83(5):1135-41. [PubMed: 16685057]

11. Kakalia S, Sochett EB, Stephens D, et al. Vitamin D supplementation and CD4 count in children infected with human immunodeficiency virus. J Pediatr. 2011; 159(6):951-7. [PubMed: 21820130] 